\title{
Modeling Smoke Visibility in CFD
}

\author{
KAI KANG \\ Hatch Mott MacDonald \\ 475 Park Avenue South \\ New York, NY 10016
}

\begin{abstract}
Smoke transport is a fundamental fire phenomenon. One area of interest in fire/life safety is computer modeling of fire and smoke transport. Smoke impairs visibility, and visibility is path-dependent. This paper presents a ray tracing technique for smoke visualization. The method of ray tracing directly addresses visibility from a viewer's perspective. A case study is performed on a t-square room fire under natural ventilation. The results demonstrate that the implemented ray tracing visualization of smoke visibility is better than other existing methods in CFD. In addition, the visualization results can be used to evaluate smoke dispersion quantitatively. For the room fire examined, the estimated standard deviation of smoke visibility helps identify the transient behavior, which occurs about $2.5 \mathrm{~min}$ to $3.5 \mathrm{~min}$ into the fire. It is expected that the better understanding of smoke visibility from this technique would benefit fire / life safety design applications.
\end{abstract}

KEYWORDS: smoke, visibility, CFD, visualization

\section{NOMENCLATURE LISTING}
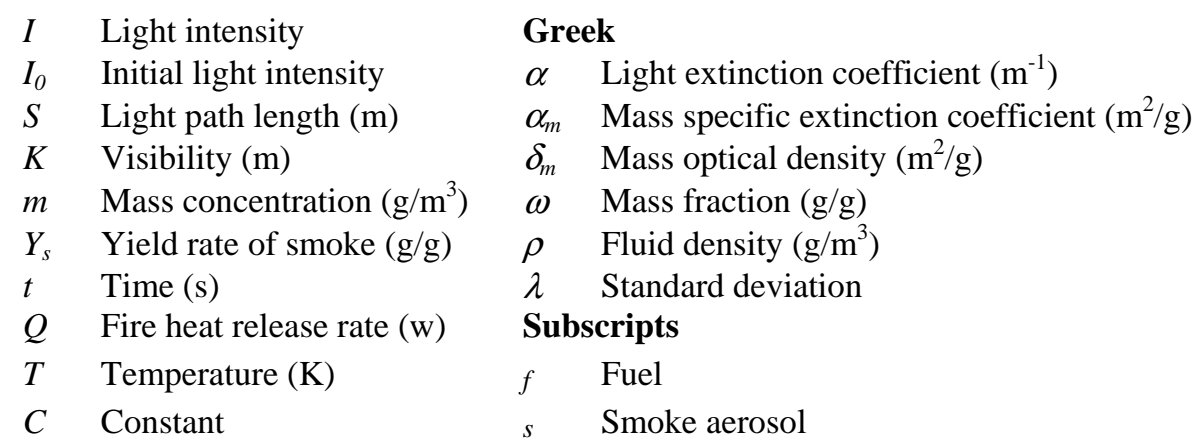

\section{INTRODUCTION}

One of the most widely measured parameters that characterize smoke aerosol is the light extinction coefficient, $\alpha$, because it directly affects visibility and smoke detection. This has been of great interest in the fire research community [1,2]. Many experiments have been done to determine smoke properties generated from flames, such as light extinction and size distribution. Various types of fuels and materials of interest to fire research have been tested $[3,4]$. Other aspects related to smoke transport, such as the toxic effects, are reported $[5,6]$. In particular, these studies generated a rich set of data on the extinction coefficients for many types of fuels at different combustion conditions. The attenuation of light penetrating a smoke layer of thickness, $S$, is governed by Bouguer's law, that is,

$$
I / I_{0}=\exp (-\alpha S)
$$


From smoke measurements, empirical formulae have been developed for phenomena associated with fire and smoke transport, such as fire heat and smoke release rate (HRR and SRR), layer interface, ceiling jet flow [7]. The US National Fire Protection Association (NFPA) adopted some of the research findings in its published design guidelines. For instance, the 2000 edition of NFPA-130 on passenger rail transit systems has included tenability provisions on smoke obscuration levels. It recommends, for smoke and fire control, to maintain a minimum $100 \mathrm{ft}(30 \mathrm{~m})$ visibility for illuminated signs at $7.5 \mathrm{ft}$ candles (80 lx) [8].

Over the years, computer modeling using computational fluid dynamics (CFD) for fire and smoke control has received increasing interest, e.g., [9]. Accurate modeling of fire and smoke flow is important, especially in the design of smoke management systems for large spaces, such as atrium [10], aircraft cargo [11], and underground mass transit systems $[12,13]$. The visibility of exit signs and escape routes is critical in the event of a fire. However, there is still a lack of tools for interpretation of smoke visibility from CFD results, which is worth further development.

The definition of visibility not only depends on smoke mass concentration, but also is a function of background contrast level and is subjective to human perception [2]. Throughout this study, the visibility is referred to as the attenuation of light or opacity, that is, the right hand expression of Bouguer's equation. Such defined visibility is more of a smoke transport property, and is independent of human light perception. Some authors have used the term of light transmission [11].

While the definition of smoke visibility depends on the line of sight, numerical modeling has not addressed it the same way as in light optical measurements. In some studies, visualization and determination of smoke visibility has relied on the mass fraction of smoke particulates, or the iso-values of computed light extinction coefficients [12]. The FDS and SmokeView program uses a "composition" technique for visualization. FDS computes soot density data on the entire 3D domain, and calculate the visibility from the empirical formula $K=C / \alpha$ [1]. It then converts and compresses this data to values used by SmokeView to draw a series of parallel, partially transparent planes [14]. This is essentially a volume composition technique but does not address visibility directly. Visibility is dependent on the line of sight, that is, path-dependent.

Given that there have been few studies on the path dependence of smoke visibility, this paper presents a simple technique, similar to ray tracing in volume rendering in computer graphics [15]. A detailed description of the developed algorithm is given first and is demonstrated through a case study on a room fire model.

\section{DESCRIPTION OF NUMERICAL MODEL ON SMOKE VISIBILITY}

\section{Light Extinction Coefficient}

In the expression of smoke visibility, the light extinction coefficient is the single most important parameter that characterizes the smoke. There are two equations that are frequently used to calculate the extinction coefficient, $\alpha$ [1,2]. The first one relates $\alpha$ with the mass optical density, $\delta_{m}$, and is given by,

$\alpha=2.303 \delta_{m} m_{f}$ 
The second equation relates the extinction coefficient with the mass specific extinction coefficient, $\alpha_{m}$, which is,

$\alpha=\alpha_{m} m_{s}$

These two equations are developed for the convenience of different experiment measurements, e.g., [1]. Ideally, these two equations should give the same value of the extinction coefficient. However, for certain type of fuel, limited experimental data might leave only one of these two equations ( $\alpha_{m}$ or $\delta_{m}$ ) applicable. The relation between the mass optical density, $\delta_{m}$, and the mass specific extinction coefficient, $\alpha_{m}$, can be found by equating these two equations,

$2.303 \delta_{m} m_{f}=\alpha_{m} m_{s}$

Rearrange for mass optical density,

$\delta_{m}=\alpha_{m} m_{s} /\left(2.303 m_{f}\right)$

Note that the smoke yield, $Y_{s}$ (g-smoke particulates / g-fuel), is defined as $Y_{s}=m_{s} / m_{f}$. Substitute $Y_{s}$ into Eq. 5, gives,

$\delta_{m}=\alpha_{m} \cdot Y_{s} / 2.303$

Equation 6 can be verified using the data available in the literature, for instance, given polystyrene ( $\mathrm{PS}, \mathrm{C}_{8} \mathrm{H}_{8}$ ), $\alpha_{m}=10.0 \mathrm{~m}^{2} / \mathrm{g}$ [3], and $Y_{s}=0.164 \mathrm{~g} / \mathrm{g}$ [1], compute the mass optical density, $\delta_{m}=10.0 \times 0.164 / 2.303=0.71 \mathrm{~m}^{2} / \mathrm{g}$. This is close to $\delta_{m}=0.79 \mathrm{~m}^{2} / \mathrm{g}$ [2] On the other hand, $\alpha_{m}$ can be calculated from Eq. 6, $\alpha_{m}=0.79 \times 2.303 / 0.164=$ $11.1 \mathrm{~m}^{2} / \mathrm{g}$, and is close to the measured value of $\alpha_{m}=10.0 \mathrm{~m}^{2} / \mathrm{g}$.

The light extinction coefficient is a function of light wavelength, e.g., the Mie theory, $\alpha$ $\propto \lambda^{-\mathrm{n}}$, where $\mathrm{n}$ is the scattering coefficient [4]. Studies have also shown that at a particular wavelength, e.g., $\lambda=633 \mathrm{~nm}$, the smoke generated by over-ventilated flame has a nearly universal specific extinction coefficient, $\alpha_{m}=8.7 \pm 1.1 \mathrm{~m}^{2} / \mathrm{g}$ with a $95 \%$ confidence interval [3]. The nearly universal constant of $\alpha_{m}$ is because of the soot formation. Note that because the soot in this case is primarily carbon spherical particles of much smaller size than light wavelength, the light extinction area per unit mass is predominantly determined by the light absorption of soot particles, and the contribution from the scattering component is small, which depends on the agglomerate size [2]. The default value of mass specific extinction coefficient in FDS is $\alpha_{m}=7.6 \mathrm{~m}^{2} / \mathrm{g}$ [14].

The convenience of Eqs. 2 and 3 is that either of the two coefficients, $\delta_{m}$ and $\alpha_{m}$, can be used in the post-processing step in CFD, particularly for $\alpha_{m}$, since it is almost an universal constant for a given light wavelength. If the soot (carbon) transport is simulated explicitly in CFD, such as in the method of volumetric heat and mass source (VHMS) [9], the extinction coefficient can be calculated from Eq. 3 for each control volume as, 


\section{Smoke visibility}

Bouguer's law is the basis for optical measurements of smoke. It relates the incident light intensity $I_{0}$, with the exiting ray intensity $I$, after a travel path length, $S$ (Eq. 1). Numerical calculation of light attenuation, the right hand of Eq. 1, requires integration along the line of sight. Because the extinction coefficient is a variable and depends on the spatial distribution of smoke concentration, Eq. 1 can be re-written in an integral form, using a finite path length, $d s$, and integrating along the line of sight, $S$, that is,

$I / I_{0}=\exp \left(-\int_{s} \alpha(s) d s\right)$

Expressed in a discretized form, gives,

$$
I / I_{0}=\exp \left(-\sum_{i} \alpha_{i} s_{i}\right)
$$

Where $s_{i}$ is the integration step. Assuming $s_{i}=s$, a constant, and $\alpha_{m}$ is a constant, substituting Eq. 7 into above, gives,

$$
I / I_{0}=\exp \left(-s \sum_{i} \alpha_{m} \rho_{i} \omega_{s, i}\right)=\exp \left(-s \alpha_{m} \sum_{i} \rho_{i} \omega_{s, i}\right)
$$

Equation 10 shows that the calculation of smoke visibility requires interpretation of mixture density $\rho_{i}$, and mass concentration of smoke aerosol $\omega_{s}$, at each integration step $i$ by "walking" along the ray. Both the mixture density and the smoke mass fraction are readily available from CFD field solutions.

\section{Algorithm Description - Volume Rendering of Smoke Visibility}

Visualization of smoke visibility defined in Eq. 1 can be obtained through ray tracing. The technique of ray tracing is well established in the computer graphics society. Forward ray tracing computes rays from light source to eye point; backward ray tracing does the opposite and is the preferred approach. If no recursive procedure for reflection or refraction of the primary ray is considered, it is also called ray casting [15]. Volume rendering using ray tracing is often associated with high computational cost, especially for unstructured control volumes (voxels). This has been discussed in a recent review on flow visualization [17]. Fruhauf et al. 1994 [18] proposed to compute ray casting in the computational domain, but the related Jacobian transformation is still expensive. Fig. 1 illustrates the concept of ray casting. A brief summary is given in the following:

- Set up camera / eye point location and orientation (Fig. 1a).

- Set up view port and its resolution.

- For each ray shoots from the eye point through each pixel center on the view port,

- Walk along the ray, and compute,

- The mixture density, and

- The mass fraction of smoke particulates. 
○ Composite transparency.

- Continue till ray terminates.

- Set proper alpha channel of the pixel and its color values (Eq. 10).

Note that the ray terminates as soon as it reaches the wall surface or any "solid" objects.

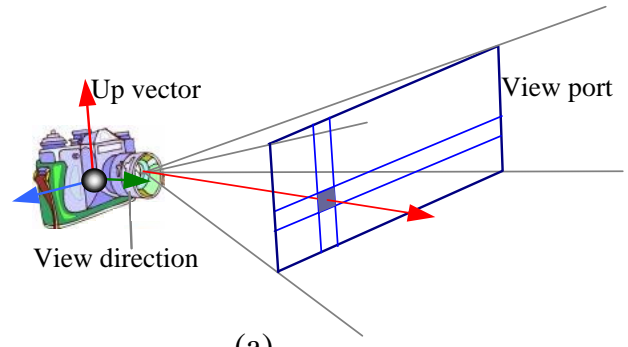

(a)

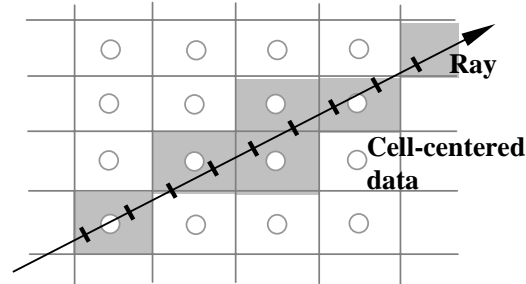

(b)

Fig. 1. Illustration of ray casting for volume rendering of smoke visibility. (a) Ray casting; (b) Integration along the ray; shaded cells indicate transverse by the ray.

To reduce the computation cost of ray tracing, the following procedures have been implemented, taking advantage of the constraints on numerical discretization.

- The first one is the use of a fixed integration step size. In CFD models, the mesh is composed of polyhedral control volumes, whose geometric aspect ratios should be close to unity. In addition, there should not be any abrupt jump in mesh sizes but rather smooth transition. Taking advantage of these mesh constraints, a constant integration step is feasible, as long as it is at least one half of the smallest mesh size, in analogy to Nyquist criteria in image re-construction.

- Numerical interpolation uses nearest cell center data, the so-called "nearest point average." This method is fast, as it avoids the time-consuming searching and rayobject intersection test. Although there are higher order interpolation schemes like tri-linear or tri-cubic Spline, the nearest point average is applicable regardless of the shape of the control volume, particularly for a hybrid mesh scheme.

- Another procedure is to separate the volume rendering data from other geometric objects. These geometric objects can be processed as surface based graphics. Effectively, the rendered scene of smoke visibility acts as an image mask to modify the "clear" scene with no smoke.

- Finally, transient CFD simulation usually gives a large dataset. Given a fixed camera location and orientation, an index table can be generated to identify the cell indices that each ray passes. Subsequent rendering can use this look-up table to quickly locate the voxels.

A flow chart of the algorithm is given in Fig. 2. It is composed of scene set up, ray tracing, and final rendering to compose the scene with smoke. The program is written in $\mathrm{C}++$ to interface with the model and the simulation data generated from a commercial CFD package, Fluent ${ }^{\circledR}$. The renderer and a graphic viewer are written using industrial standard OpenGL API v1.2 [19]. The image is composed in RGBA format, and visibility is obtained by controlling the pixel transparency / opacity through alpha channel blending. The rendering is entirely color-based. This eliminates subjective factors, such 
as lighting, background contrast, and human perception, and is consistent with the definition of smoke visibility discussed at the beginning of this paper.

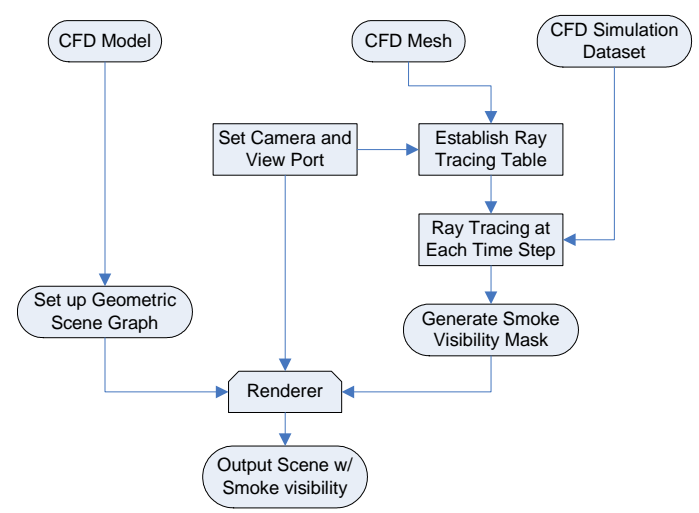

Fig. 2. Flowchart of ray tracing rendering of smoke visibility.

\section{ROOM FIRE}

\section{Description of the Fire Scenario}

To illustrate this technique, a CFD model is set up for a simple room fire. Fig. 3a shows the dimension of the room. It has a square floor area $30 \mathrm{~m} \times 30 \mathrm{~m}$, and the ceiling height is $10 \mathrm{~m}$. There are three doors on one sidewall and three window openings on the opposite sidewall (Fig. 3a). In the ceiling area near the doorway, a drop ceiling of $3 \mathrm{~m}$ deep is used as a passive smoke barrier. Due to the room's high ceiling, only natural ventilation is considered. The doors and windows are modeled as openings to ambient. All the sidewalls, including floor and ceiling, are assumed to be adiabatic. This model can be related to a shopping mall or a warehouse.

The fire $H R R$ follows a t-square curve, defined as $Q=43 \times t^{2}$. In the simulation, it grows to about $3 \mathrm{MW}$ in 265 seconds. The fuel is assumed to be polystyrene (PS, $\mathrm{C}_{8} \mathrm{H}_{8}$ ), and its heat of combustion is assumed to be $39.2 \mathrm{MJ} / \mathrm{kg}$. Fire is located in the center of the floor defined as a volume $W \times L \times H=1.0 \mathrm{~m} \times 1.0 \mathrm{~m} \times 0.5 \mathrm{~m}$, and is modeled using the volumetric heat and mass source (VHMS) method [9]. The VHMS method only considers the transport of reactants and products from the combustion process, given by,

$\mathrm{C}_{8} \mathrm{H}_{8}+7.42\left(\mathrm{O}_{2}+3.76 \mathrm{~N}_{2}\right) \rightarrow$

$5.51 \mathrm{CO}_{2}+3.59 \mathrm{H}_{2} \mathrm{O}+0.09 \mathrm{C}_{8} \mathrm{H}_{8}+0.22 \mathrm{CO}+0.11 \mathrm{CH}+1.42 \mathrm{C}(\mathrm{s})+27.9 \mathrm{~N}_{2}$

Where the yield rate of each chemical compound is obtained from [1] and [2], and smoke is assumed composed mostly of carbon particles, whose yield rate is $0.164 \mathrm{~g} / \mathrm{g}$ [1]. For smoke visibility calculation, the light specific extinction coefficient, $\alpha_{m}=10 \mathrm{~m}^{2} / \mathrm{g}$ [3] is used in Eq. 10.

CFD solution procedure follows that in the numerical validation study on a compartment fire [9]. For turbulence, standard $k-\varepsilon$ model is modified with buoyancy term included in both equations. For transient solution, the time step is limited to $5 \mathrm{~s}$ or less and the results 
are saved every $5 \mathrm{~s}$. Convergence criteria for the residuals are set to be $10^{-6}$ for energy and smoke, e.g., carbon, and $10^{-3}$ for continuity, momentum, and other species.

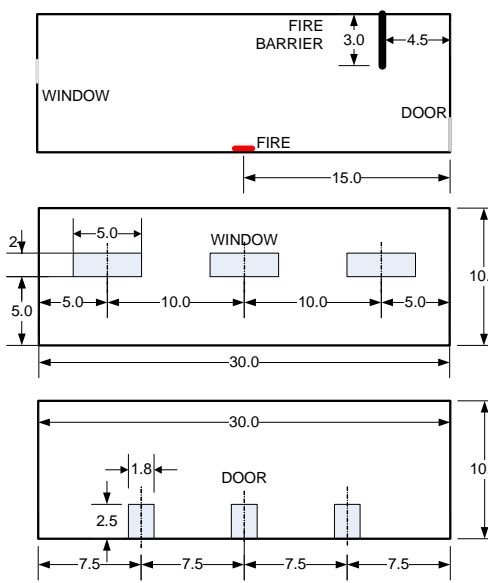

(a)

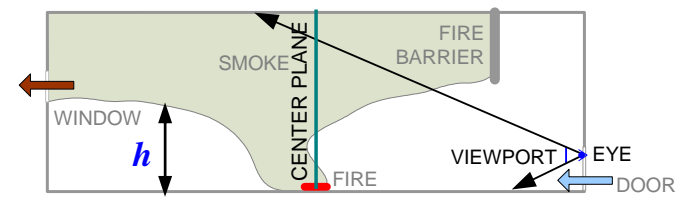

(b) Mid-plane

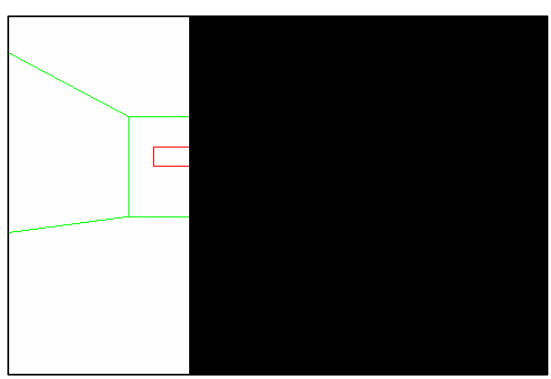

(c) Room rendered in view port

Fig. 3. (a) Room fire model (mid-plane; window layout; and door layout);

(b) Mid-plane; and (c) View port rendered scene.

\section{Visualization Set-up}

It is expected that the smoke distribution in the middle section of the room would be similar to a compartment fire. The buoyancy-driven flow is depicted in Fig. 3b with the make-up air comes through the door openings and the smoke and hot gas from the fire leave through the windows. The smoke is expected to form two zones of distinctive layers of smoke mass concentration and visibility.

Figure 3b also shows the visualization set up. The camera / eye location is in the centerline of the middle door and has a $2 \mathrm{~m}$ elevation from the floor. The view port is positioned $1 \mathrm{~m}$ from the eye point and is set as $W \times H=2.4 \mathrm{~m} \times 1.6 \mathrm{~m}$, giving a field of view angle of about $60^{\circ}$. Based on the view port of $240 \times 160$, a total of 38,400 rays from the eye point are traced through the scene in a scan conversion fashion [15]. There is no lighting in the scene, or the initial light intensity is the same regardless of the position in the room. Figure $3 c$ shows the rendered interior of the room, in which the three windows are represented by the three small rectangles. These windows are $5 \mathrm{~m}$ above the floor, which will be used as a reference in the next section to examine the results.

\section{RESULTS AND DISCUSSION}

\section{Visualization of Smoke Visibility}

The visualization of smoke visibility is shown in Fig. 4 for the initial 5 min of fire. The darkness of the visibility in Fig. 4 is based on the "opacity", which is between 0 and 1, with 0 being completely transparent and 1 being completely opaque. It shows the change of visibility conditions inside the room as a result of smoke dispersion near the ceiling 
and the sidewalls. The middle window, which is behind the fire, becomes invisible after $2 \mathrm{~min}$. At $\mathrm{t}=2 \mathrm{~min}$, Fig. 4c also indicates that the smoke start to descend from the ceiling, and reaches the floor on the perimeter of the room. As time progresses, reasonable visibility of $30 \mathrm{~m}$ cannot be kept within $5 \mathrm{~m}$ above the floor from the $2^{\text {nd }}$ min to the $3^{\text {rd }}$ min. Fig. 4 e shows all the windows are mostly invisible and Fig. $4 \mathrm{f}$ shows only partially visible windows.

It should be noted that visibility is a "bi-direction" parameter. In this case, the camera is at the center door (Fig. 3b). If the camera cannot see a certain area inside the room, it also means that for anybody "trapped" in that area of the room, he / she cannot see the center door as well.

Figure 4d shows a much worse condition than that in Fig. 4f. However, Fig. 4f is of a later time at $\mathrm{t}=240 \mathrm{~s}$, when the $H R R$ more than doubles that at $\mathrm{t}=160 \mathrm{~s}$, that is, $2.5 \mathrm{MW}$ vs. 1.1 MW. This could be due to the transient behavior before the flow pattern is fully established. The room air volume will expand during the initial few minutes because of the heat from the fire, and the mass inside the room will not be constant. It takes the system some time to establish the buoyancy driven flow (Fig. 3b) due to fluid inertia.

What is of the most interest to fire/life safety is the clear definition of the boundary of "dense" smoke as visualized in Fig. 4. Such phenomenon is similar to what has been observed in fire testing. However, the smoke "boundaries" cannot be easily identified through conventional surface based graphics, for example, the contour of the smoke mass fraction. Two contour plots of smoke mass fraction of the center plane are shown in Fig. 5 at $t=180 \mathrm{~s}$ and $t=240 \mathrm{~s}$. Figures $5 \mathrm{a}$ and $5 \mathrm{~b}$ are different, but the visibility conditions shown in Figs 4e and $4 \mathrm{f}$ do not seems that different. Indeed, Fig. 4e is slightly worse than Fig. $4 \mathrm{f}$ with respect to clearance height. The surface-based representations are not directly applicable to determine visibility conditions. This is because the mass fraction distribution is a spatial variable, and the visibility is an "integral" parameter.

\section{Quantification of Visibility}

A quantitative evaluation of smoke visibility can be obtained from the image standard deviation, $\lambda=\sqrt{\sum_{i}\left(I_{i}-\bar{I}\right)^{2} /(N-1)}$ where $I_{i}$ is each pixel's opacity, and $\bar{I}$ is the average opacity of all pixels, and $N$ is the total number of pixels. The standard deviation evaluates the uniformness of an image's darkness. In this case, $\lambda=0$ means no smoke; and the increase in standard deviation suggests increased dispersion of smoke.

Figure 6a is a plot of the standard deviation as a function of time. The transient behavior is indicated by the curve's fluctuation. The standard deviation, $\lambda$ reaches the maximum (Point " $\mathrm{A}$ ") at $\mathrm{t}=135 \mathrm{~s}$, when almost $50 \%$ of the view port is obscured. It then reduces to Point "B" at $t=200 \mathrm{~s}$, before climbing back with a much slower rate. Corresponding smoke mass fraction contours in Fig. $6 \mathrm{~b}$ indicate that $\mathrm{t}=200 \mathrm{~s}$ is worse than $\mathrm{t}=135 \mathrm{~s}$, while the visibility from the "eye" point (Figs. 4d and 4e) seems to be the opposite.

\section{Discussion}

Ray tracing rendering of smoke visibility is able to reveal quantitatively such transient response. The transient behavior is due to fluid inertia, that is, the system takes time from initial tranquil condition to the established buoyancy driven flow (Fig. 3b). However, in 
the CFD model, the fire $H R R$ growth rate is imposed as a t-square curve, while in reality, the fire growth is rather a dependent variable on fuel mass transfer, turbulent mixing and oxygen availability; that is, the so-called ventilation controlled fire. The transient behavior is still worth further experiment validation.

On the other hand, ray-tracing rendering of smoke visibility allows for direct comparison and validation of CFD models with results from light optical measurements [3]. For example, the comparison on light transmission in an aircraft cargo compartment fire used a similar approach [11]. This technique can also be modified to incorporate the smoke aerosol size distribution, such as the scattering contribution from numerical modeling of soot coagulation [14]. Additionally, ray tracing has already been implemented as part of the radiation sub-models in CFD. The discrete transfer radiation model (DTRM) [16] solves a simplified version of the radiation transfer equation,

$$
\frac{d I}{d s}+a I=\frac{a \sigma T^{4}}{\pi}
$$

The DTRM integrates this equation along a series of pre-defined rays emanating from boundary faces. If ignoring the right hand, and integrating along a ray, $S$, it is then,

$$
I=I_{0} \exp \left(-\int_{S} a \mathrm{ds}\right)
$$

This is exactly the same form as Eq. 8. The outlined ray-tracing algorithm can thus be integrated as part of post-processing in CFD, utilizing the DTRM solver.

One area of practical application is in the evaluation of smoke management systems, such as for underground mass transit [8]. The CFD study [12] used surface contours of extinction coefficient to interpret visibility. Another study [13] reported the spatial distribution of visibility, determined from $K=C / \alpha$, which is similar to that in FDS [14]. While these studies have been able to predict the smoke flow, the visibility conditions are not addressed directly from a viewer's perspective. Current work is underway to apply this algorithm in fire life safety applications. Future investigations should include validation of the predicted visibility with experimental results.

\section{CONCLUSIONS}

This paper presents a ray tracing approach on visualization of smoke visibility in CFD. Smoke visibility is defined as an exponential decay of light transmission through smoke layers, which eliminates other subjective factors, such as lighting and perception. The developed ray-tracing algorithm expedites the computation by taking advantage of the CFD mesh constraints, and by separating volume rendering data from the surface based geometric objects.

The developed technique is applied to a room fire under natural ventilation. The smoke migration inside the room is visualized based on the numerical results. Comparisons are made to demonstrate the deficiency of conventional surface contours to determine visibility. The visualization result is able to clearly identify the smoke "boundary". Such result cannot be obtained by setting a threshold value on smoke concentration. In addition, for the room fire considered here, the transient behavior is evaluated by the 
standard deviation of smoke visibility in rendered images. The transient behavior is due to fluid inertia for the establishment of the buoyancy-driven flow pattern. It happens within the first 3 min to 4 min into the fire. The standard deviation peaks at about $2.5 \mathrm{~min}$ into the fire, and then decreases for 1 min before slowly increasing again. The approach presented in this paper addresses visibility directly from a viewer's perspective, which is important in practical fire / life safety design.

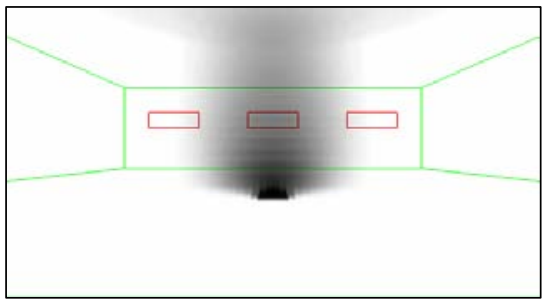

(a) $\mathrm{t}=30 \mathrm{~s}, \mathrm{Q}=39 \mathrm{~kW}$

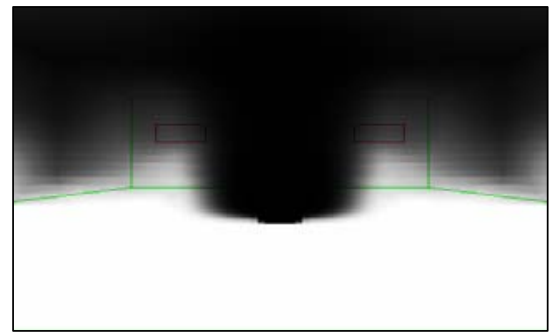

(c) $\mathrm{t}=120 \mathrm{~s}, \mathrm{Q}=0.6 \mathrm{MW}$

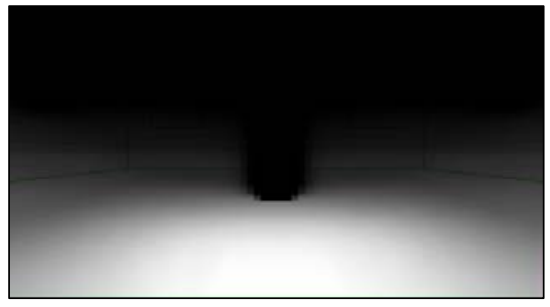

(e) $\mathrm{t}=180 \mathrm{~s}, \mathrm{Q}=1.4 \mathrm{MW}$

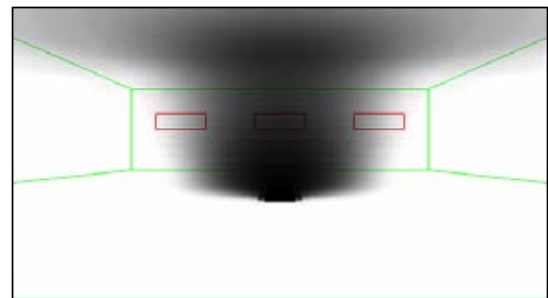

(b) $\mathrm{t}=60 \mathrm{~s}, \mathrm{Q}=155 \mathrm{~kW}$

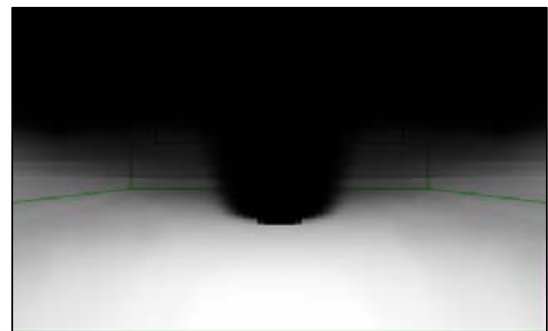

(d) $\mathrm{t}=160 \mathrm{~s}, \mathrm{Q}=1.1 \mathrm{MW}$

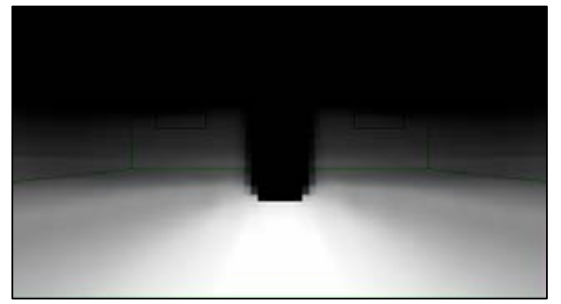

(f) $t=240 \mathrm{~s}, \mathrm{Q}=2.5 \mathrm{MW}$

Fig. 4. Visualization of smoke visibility at typical time during the fire growth.

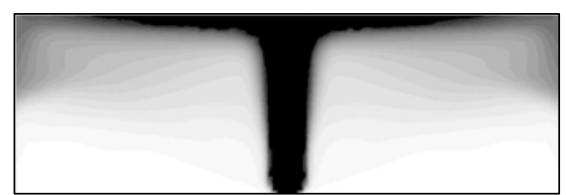

(a) $\mathrm{t}=180 \mathrm{~s}, \mathrm{Q}=1.4 \mathrm{MW}$

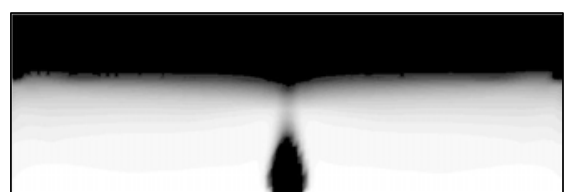

(b) $\mathrm{t}=240 \mathrm{~s}, \mathrm{Q}=2.5 \mathrm{MW}$

Fig. 5. Distribution of smoke mass fraction in the center plane of the room. 


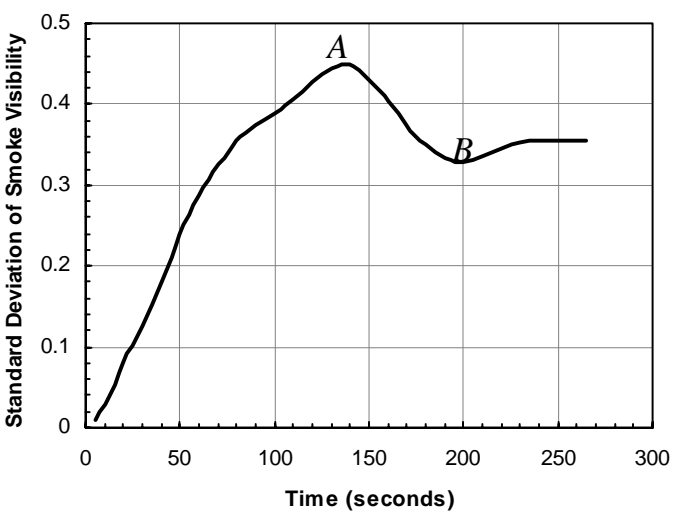

(a)
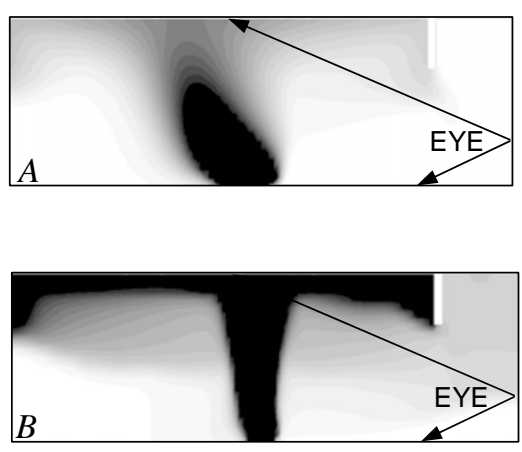

(b)

Fig. 6. (a) Standard deviation of the view port smoke visibility as a function of fire growth time; (b) Smoke mass fraction distribution in the mid-plane at selected times frames (A: $t=135 \mathrm{~s} ; \mathrm{B}: \mathrm{t}=200 \mathrm{~s})$.

0.0

5e-5

\section{REFERENCES}

[1] Klote J.H., and Milke, J.A., Design of Smoke Management Systems, American Society of Heating, Refrigerating, and Air-conditioning Engineers, Atlanta, GA, 1992.

[2] Mulholland, G.W., "Smoke Production and Properties," The SFPE Handbook of Fire Protection Engineering, $2^{\text {nd }}$ Ed., DiNenno P.J. (ed.), National Fire Protection Association, Quincy, MA, 1995, p. 217-227.

[3] Mulholland, G.W., and Croarkin, C., "Specific Extinction Coefficient of Flame Generated Smoke,” Fire Mater., 24, pp. 227-230, (2000).

[4] Barakat, M., Souil, J.M., Breillat, C., Vantelon, J.P., Knorre, V., and Rongere, F.X., "Smoke Data Determination for Various Types of Fuel," Fire Safety J., 30, pp. 293-306, (1998).

[5] Bulter, K.M., and Mulholland, G.W., "Generation and Transport of Smoke Components,” Fire Technology, 40, pp. 149-176, (2004).

[6] Gann, R.G., "Sublethal Effects of Fire Smoke,” Fire Technology, 40, pp. 95-99, (2004).

[7] Peacock, R.D., and Babrauskas, V., "Analysis of Large-scale Fire Test Data," Fire Safety J., 5, pp. 387-414, (1991).

[8] NFPA-130 Standard for Fixed Guideway Transit and Passenger Rail Systems, 2000 Edition, National Fire Protection Association, Quincy, MA, 2000.

[9] Kang, K., “Computational Study of Longitudinal Ventilation Control of Tunnel Enclosure Fire,” submitted to J. of Fire Protection Engineering. 
[10] Klote, J.H., “An Overview of Atrium Smoke Management,” J. of Fire Protection Engineering, 7, pp. 24-34, (2002).

[11] US DOT / FAA, “Computational Fluid Dynamics Code for Smoke Transport during an Aircraft Cargo Compartment Fire: Transport Solver, Graphical User Interface, and Preliminary Baseline Validation,” Office of Aviation Research, Washington, DC, 2003, 31 p.

[12] Zigh, A., Ong, I., and Kang, K., "CFD Applications for Subway Stations," Proceedings of the 10th International Conferences on Aerodynamics and Ventilation of Vehicle Tunnels, Boston, MA, 2000.

[13] Shahcheraghi, N., McKinney, D., and Miclea, P., "Effect of Fan Start Time on Emergency Ventilation System,” Tunnel Management International Journal, 6 (4), 2003.

[14] McGrattan, K.B., Forney, G.P., Prasad, K., Floyd, J.E., and Hostikka, S., "Fire Dynamics Simulator (version 3) - User’s Guide,” National Institute of Standards and Technology report NISTIR 6784, Gaithersburg, MD, 2002, 61 p.

[15] Foley, J., Van Dam, A., Feiner, S., and Hughes, J., Computer Graphics Principles and Practices, Addison Wesley, 1990.

[16] Carvalho, M.G., Farias, T., and Fontes, P., "Predicting Radiative Heat Transfer in Absorbing, Emitting, and Scattering Media Using the Discrete Transfer Method," Fundamentals of Radiation Heat Transfer, Fiveland et al. (ed.), (1991), 160, pp. 17-26, ASME HTD.

[17] Crawfis, R.A., Shen, H.W., and Max, N., "Flow Visualization Techniques for CFD Using Volume Rendering," Proceedings of the 9th International Symposium on Flow Visualization, 2000.

[18] Fruhauf, T., "Raycasting of Nonregularly Structured Volume Data," EUROGRAPHICS'94, Daehlen, M., and Kjelldahl, L. (ed.), Blackwell Publishers, 13, (3), 1994.

[19] Woo, M., Neider, J., Davis, T., and Shreiner, D., OpenGL Programming Guide: the Official Guide to Learning OpenGL, Version 1.2, OpenGL Architecture Review Board, ( $3^{\text {rd }}$ Ed.), Addison-Wesley, MA, 1999.

[20] Farouk, B., Mulholland, G.W., McGrattan, K.B., and Cleary, T.G., "Simulation of Smoke Transport and Coagulation for a Standard Test Fire," Proceedings of the 12th International Conference on Automatic Fire Detection, NIST, Gaithersburg, MD, 2001. 